\title{
Las bibliotecas de ciencias de la salud del Sistema Sanitario Regional de Madrid
}

\begin{abstract}
Resumen: Las bibliotecas de ciencias de la salud del Sistema Regional Sanitario de Madrid iniciaron en 2002 un proyecto de coordinación y cooperación bibliotecaria. En el presente trabajo se exponen los antecedentes de esta situación (culminación del proceso de transferencias sanitarias, creación de la "Agencia Laín Entralgo para la Formación, Investigación y Estudios Sanitarios" adscrita a la Consejería de Sanidad, y creación en el seno de dicha Agencia del "Comité de Bibliotecas de Ciencias de la Salud”. Además, se exponen algunos de los logros de esta experiencia consorciada (colección electrónica compartida de recursos electrónicos, programa de formación de usuarios y biblioteca virtual).
\end{abstract}

Palabras clave: Consorcios bibliotecarios, Bibliotecas de ciencias de la salud, Madrid.

\section{Title: The health science libraries of the Regional Health System of Madrid}

Abstract: This library co-operation and co-ordination project was started in 2002 by the health libraries of the Regional Health System of Madrid. Its background -including the completion of the transfer of health responsibilities from the central government to the autonomic government, the creation of the "Lain Entralgo Agency for Training, Research and Health Studies" as well as the creation within the Agency of the "Committee of Health Libraries"-are described in this paper. Furthermore, the achievements of this consortium (sharing electronic collections, common patron training programmes and digital library) are also explained.

Keywords: Library consortia, Health libraries, Madrid. 


\section{Antecedentes}

LAS BIBLIOTECAS DE CIENCIAS DE LA SALUD en España han reiterado con frecuencia la ineludible necesidad de una cooperación y coordinación bibliotecaria desde la celebración en Santander, en 1986, de sus primeras jornadas de ámbito nacional. El primer resultado concreto de esta cooperación fue la elaboración del Catálogo colectivo de publicaciones periódicas en bibliotecas de ciencias de la salud españolas, publicado en formato impreso en 1996 y accesible en internet desde el año 2000. En 1998, y en un escenario no exclusivamente sanitario como fue la Reunión de consorcios bibliotecarios en Cádiz, comenzó a plantearse con mayor rigor entre las bibliotecas de salud la necesidad de crear nuevas estructuras de cooperación bibliotecaria en las diferentes comunidades autónomas.

Así, en 2000, las bibliotecas de ciencias de la salud de los hospitales de Madrid junto con la de la Consejería de Sanidad presentaron a la Dirección Provincial del Insalud un proyecto de creación de un consorcio de bibliotecas de ciencias de la salud que no llegó a ser viable ante el inminente proceso de ejecución de las transferencias sanitarias.

La culminación de dichas transferencias desde el gobierno central al autonómico determinó un nuevo panorama organizativo regido por la entrada en vigor de la Ley 12/2001, de 21 de diciembre, de Ordenación sanitaria de la Comunidad de Madrid (Loscam). El título $\mathrm{X}$ se refiere específicamente a la creación de la Agencia Laín Entralgo para la formación, investigación y estudios sanitarios, adscrita a la Consejería de Sanidad, que se articula como un instrumento para el desarrollo de la política de formación e investigación sani- tarias, la coordinación y evaluación de programas con otras entidades e instituciones que actúan en este ámbito, y el apoyo al sistema sanitario de la Comunidad de Madrid.

Con el fin de atender las necesidades de información bibliográfica y documentación científica de los profesionales de la salud se crea, dependiente de la Agencia Lain Entralgo, el Centro de Información Bibliográfica y Documentación en Ciencias de la Salud. En el artículo 127 de la Loscam quedan definidas sus funciones:

-facilitar y agilizar el acceso a la información y documentación científica;

- gestionar las suscripciones y administrar los servicios de biblioteca y hemeroteca; $y$

— procurar la colaboración y el establecimiento de acuerdos y convenios interbibliotecarios.

\section{Comité de Bibliotecas de Ciencias de la Salud de la Agencia Laín Entralgo}

Para llevar a cabo la realización de las funciones del Centro de Información Bibliográfica y Documentación, se constituye el Comité de profesionales representantes de las bibliotecas de ciencias de la sa$l u d$, recogido en el Decreto 139/2002, de 25 de julio, el mismo en el que se establece el régimen jurídico y de funcionamiento de la agencia. Son funciones de este comité:

- la coordinación de las bibliotecas de ciencias de la salud, optimizando los recursos existentes y ofreciendo servicios de valor añadidos;

—el diseño y planificación de la gestión de la información, gestión de contenidos y gestión del conocimiento, mediante la aplicación de nuevas tecnologías, facilitando un entorno común de trabajo tanto a usuarios como a profesionales, orientado a la mejora de la calidad de los servicios y a la optimización de los costes;

—el desarrollo de la normativa de funcionamiento del comité, procedimientos técnicos y servicios de las bibliotecas de ciencias de la salud;

- la propuesta de acuerdos y convenios de ámbito nacional e internacional con instituciones tanto públicas como privadas, que permitan ampliar el entorno del conocimiento y difundirlos a toda la comunidad científica; y

—el diseño y propuesta de programas de formación continuada para los usuarios y profesionales de las bibliotecas de ciencias de la salud.

En este marco, las bibliotecas presentaron un nuevo proyecto de consorcio bibliotecario a los responsables de la Agencia Laín Entralgo en el que se incluyó un análisis detallado de su situación (estudio de las colecciones de revistas científicas impresas y bases de datos, accesos electrónicos, costes, usuarios reales y potenciales, infraestructuras informáticas y de comunicaciones, procesos y procedimientos de trabajo, etc.), así como un estudio de los requerimientos mínimos para la creación de una biblioteca electrónica compartida (definición de una red única informática, líneas de comunicaciones, software de gestión, recursos humanos, programa de formación, definición de una colección electrónica compartida de recursos de información científica, diseño de un entorno web, y creación de grupos de trabajo para el desarrollo de las funcionalidades de este entorno).

La Agencia Laín Entralgo estudió la viabilidad del proyecto de consorcio presentado por las bibliotecas, desestimándolo como modelo de asociación ya que, asu- 


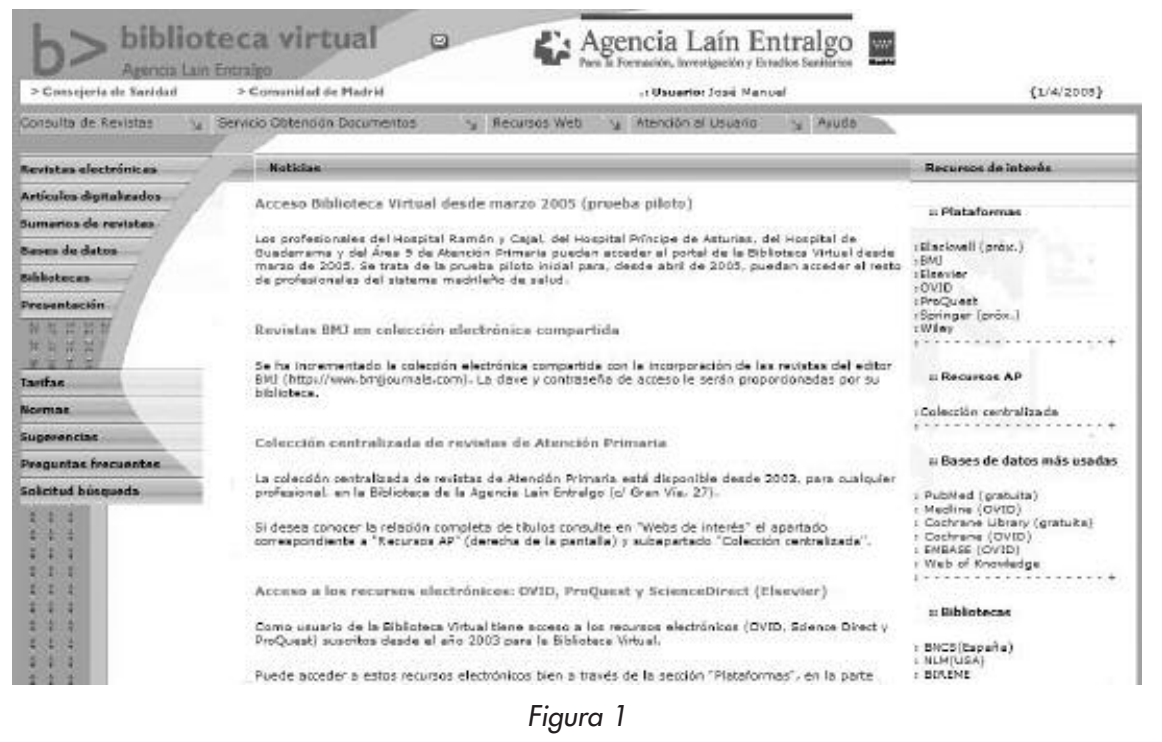

midas las transferencias sanitarias, todos los centros hospitalarios (donde se ubican las distintas bibliotecas) estaban adscritos a una misma autoridad, la Consejería de Sanidad. En consecuencia, se estableció la creación del comité como forma jurídica viable de cooperación interbibliotecaria.

En la actualidad, están representadas en el comité, a través de sus responsables o directores/as, la biblioteca de la Agencia Laín Entralgo y las bibliotecas de todos los hospitales del sistema público sanitario de Madrid. Para su desarrollo cooperativo, en el seno del comité se han constituido diferentes grupos de trabajo (publicaciones periódicas, préstamo interbibliotecario, difusión selectiva de la información, formación, etc.) que evalúan las actividades desarrolladas, proponen otras para su cumplimiento y presentan un resumen de su cometido en las reuniones periódicas del comité.

\section{Dos años de cooperación y coordinación bibliotecaria}

La total implicación institucional ha supuesto la definición de una política clara en la toma de decisiones para la ejecución de todos los objetivos del proyecto. La Agencia Laín Entralgo ha asumido la gestión económica, que incluye fica, posibilitando su acceso de forma ágil, autónoma y en un entorno remoto (figura 1);

-el diseño e implementación de un Servicio de Obtención de Documentos (SOD) automatizado;

- la creación de un programa anual de formación continuada en documentación científica y recursos de información para todos los profesionales sanitarios de Madrid;

—el desarrollo de un programa de formación continuada específico para los profesionales de las bibliotecas del comité;

- la difusión de los contenidos de la colección electrónica compartida en catálogos y bases de datos en entorno web (C-17 o PubMed-Linkout); y

- el estudio estadístico de usuarios sobre el acceso y utilización de los recursos electrónicos de información científica.

\section{Biblioteca Virtual Agencia Laín Entralgo}

Desde 2003 los profesionales mité:

Todo ello ha permitido al co-

- el establecimiento de una política de racionalización de la colección impresa de revistas científicas de todas las bibliotecas;

- la definición y desarrollo de una colección compartida de revistas científicas electrónicas (1.248 títulos, accesibles a través de OVID-Lippincott, ProQuest, ScienceDirect y Wiley);

- la selección de un conjunto de bases de datos y fuentes de información (Medline, Cochrane Library, Embase Drugs \& Pharmacology, Embase Psychiatry, Pascal y Cinahl, entre otras).

—el diseño e implementación de un entorno digital único (biblioteca virtual) en el que se han integrado los servicios bibliotecarios y los recursos de información cientí- sanitarios han venido accediendo a los recursos electrónicos de información científica desde las propias plataformas de los editores, y no ha sido hasta la primavera de 2004 cuando la Biblioteca Virtual se ha puesto en producción (en esta primera fase sólo para el personal bibliotecario), permitiendo desde entonces la actualización de los catálogos, la activación del préstamo interbibliotecario y la redefinición de algunas funcionalidades.

El acceso a la plataforma de servicios de la Biblioteca Virtual por parte del usuario no se ha producido hasta enero-febrero de 2005. En una primera fase piloto se ha facilitado el acceso sólo a un determinado número de usuarios pertenecientes a unos centros seleccionados como muestra, para así validar la puesta en producción de todas las funcionalidades del siste- 
ma y dimensionar la disponibilidad de la red de comunicaciones. Con posterioridad se ha ido proporcionando el acceso, de forma progresiva, al resto de los usuarios, hasta un total de 20.000. Para su puesta en marcha se ha diseñado un cursotaller de biblioteca virtual que, con una duración de 3 horas, pretende dar a conocer los distintos servicios bibliotecarios que ofrece y facilitar el aprendizaje y manejo de los nuevos recursos electrónicos disponibles.
Actualmente todos los esfuerzos están centrados en la consecución de varios objetivos: dinamizar los procesos, establecer acciones de mejora en el sistema ejerciendo una evaluación permanente que permita detectar y subsanar fallos, ampliar la cobertura de la colección electrónica compartida a partir del estudio estadístico del uso de la colección y de las necesidades de los usuarios, y proseguir con el desarrollo de una política de racionalización de las colecciones impresas.
El proceso de transición a la biblioteca electrónica es ya en una realidad entre las bibliotecas del Sistema Regional Sanitario de Madrid y definitivamente se evidencia como irreversible ante el crecimiento progresivo de la demanda y las expectativas de los usuarios.

Comité de Bibliotecas de Ciencias de la Salud de la Agencia Laín Entralgo, Agencia Laín Entralgo.

C/ Gran Vía, 27, Madrid 28013.

bibliotecavirtual@salud.madrid.org 